Article

\title{
Computational Assessment of Modified Antifreeze Glycoproteins on Ice Nucleation
}

\author{
Monika Bleszynski *(D) and Matt Reil \\ Department of Mechanical \& Materials Engineering, Ritchie School of Engineering and Computer Science, \\ University of Denver, Denver, CO 80208, USA; matt.reil@du.edu \\ * Correspondence: mbleszyn@du.edu; Tel.: +1-720-545-4075
}

Citation: Bleszynski, M.; Reil, M. Computational Assessment of Modified Antifreeze Glycoproteins on Ice Nucleation. Biophysica 2021, 1 , 168-178. https://doi.org/10.3390/ biophysica1020013

Academic Editor: Serge Perez

Received: 2 April 2021

Accepted: 3 May 2021

Published: 6 May 2021

Publisher's Note: MDPI stays neutral with regard to jurisdictional claims in published maps and institutional affiliations.

Copyright: (c) 2021 by the authors. Licensee MDPI, Basel, Switzerland. This article is an open access article distributed under the terms and conditions of the Creative Commons Attribution (CC BY) license (https:// creativecommons.org/licenses/by/ $4.0 /)$.

\begin{abstract}
Antifreeze glycoproteins (AFGPs) found in various fish are used by the organisms to prevent freezing. While these compounds have been studied for their ability to bind to, and prevent the complete crystallization of water, the exact mechanisms by which AFGPs prevent freezing are still undetermined. Therefore, building upon our previous work, this study uses molecular dynamics simulations to assess the effects of hydroxyl group separation distance on AFGP ice nucleation activity. Water droplet crystallization simulations showed that modified AFGP structures containing hydroxyl distances smaller than $\sim 3.0 \AA$ lost their ability to prevent ice crystallization. Furthermore, modified AFGP containing hydroxyl distances of $7.327 \AA$ and $6.160 \AA$ was correlated with a promotion in ice nucleation, as demonstrated by the changes in the energy of the system. This supports the notion that the distance, and therefore, geometry characteristics between the hydroxyl groups located on the saccharide structures play a key role in the ice crystallization inhibition properties of AFGP compounds.
\end{abstract}

Keywords: antifreeze glycoproteins; molecular dynamics; ice nucleation; hydroxyl group separation distance

\section{Introduction}

Various antifreeze compounds have been identified in plants, insects, and fish that hold the ability to resist ice formation, allowing them to survive in cold weather and subfreezing waters [1-5]. These antifreeze compounds are often termed ice-binding proteins (IBPs) to denote that their primary mechanism for preventing ice formation occurs by adhering to ice crystal planes, though this may vary by IBP type [1-7].

Compounds that can depress the hysteresis point of water are often termed antifreeze proteins (AFPs), as well as antifreeze glycoproteins (AFGPs) [2-4]. AFGP compounds from the Antarctic Notothenioids and other fishes have been studied in depth to better understand exactly how the glycoprotein interacts with water molecules to prevent complete crystallization [3-7]. Various studies have assessed the effects of molecular weight on ice crystallization, while others have focused on the peptide structure and length [5-16]. Further studies have found that the structure itself determines ice-binding properties, and that AFGPs that take on an $\alpha$-helix structure cannot bind to ice [17].

Likewise, some studies have examined the effects of the sugar structures on ice nucleation both jointly and independently with the peptide structure, to better understand the roles that each structure plays in preventing crystallization [1-12]. For example, Pandey et al. utilized molecular dynamics to observe the effects of four key structures that were determined to be critical to AFGP activity, one of which was determined to be the presence of the structure's carbohydrate hydroxyl groups [3-11].

However, there are still many unanswered questions regarding whether the peptide or the saccharide structure specifically is responsible for the antifreeze properties of AFGPs, and there are conflicting hypotheses regarding which structure is more responsible for 
the molecular mechanism of ice-binding, and thus, AFGP antifreeze activity [6]. For example, some studies have found that activity is mainly due to the hydrophobic peptide backbone $[3,4,7,8,13]$. In contrast, others have found the hydrophilic saccharide groups to be primarily responsible for interacting with water during crystallization [9]. Yet other studies have found the threonyl residue in the tripeptide unit to be the significant component responsible for antifreeze, while finding the overall saccharide structure to be non-essential [11]. Thus, the exact mechanisms by which AFGPs bind to ice and prevent complete crystallization have not been thoroughly determined, and there are still several conflicting hypotheses.

Nevertheless, the sugar moieties are at least partially responsible for inhibiting ice crystallization, and these structures have been investigated by numerous studies [3-11]. Some have suggested that AFGPs have significant variability in their ice-binding and crystallization inhibiting behavior even if the sugar structures are only slightly modified. For example, Ahn et al. investigated the effects of increasing the number of hydroxyl groups on the saccharide structure. Their study found that despite increasing the number of essential hydroxyl groups on the structures, no enhanced antifreeze activity was reported for their structures, indicating that the antifreeze activity relies on both the presence and intrinsic conformations of the hydroxyl groups [11].

We previously investigated how the hydroxyl distances in modified poly(vinyl) alcohol (PVA) polymers affect ice nucleation [18]. Using molecular dynamics simulations, we found that PVA compounds with a specific hydroxyl distance range were associated with nucleation inhibition (NI), while other hydroxyl distances were correlated with the radial distribution between the $\mathrm{O}_{\text {ice }}-\mathrm{O}_{\text {ice }}$. Thus, we concluded that for ice nucleation inhibition (NI) to occur, the hydroxyl distance (HD) in PVA compounds must be greater than $2.858 \AA$, but less than $7.117 \AA$ [18]. Similarly, the hydroxyl distances in common antifreeze compounds, such as ethylene glycol and propylene glycol, are within this range. When measured using molecular dynamics software, the hydroxyl distances for ethylene glycol and propylene glycol, measured from $\mathrm{O} \cdots \mathrm{O}$, are $3.832 \AA$ and $2.911 \AA$, respectively. Likewise, the measured hydroxyl distances in AFGP-8 range between $\sim 3.455 \AA$ to $\sim 5.518 \AA$. Previous studies have shown that molecular dynamics simulations are an effective tool for understanding how antifreeze compounds bind to and interact with ice as it nucleates [19-24]. Building upon this data, this study uses molecular dynamics simulations to investigate how the hydroxyl distances on the sugar moieties of AFGP may affect its ice nucleation inhibition and ice-binding properties.

\section{Materials and Methods}

To better understand if hydroxyl group separation distance affects the ice-binding and nucleation inhibition properties of AFGP compounds, a series of molecular dynamics simulations of a freezing water droplet were performed. Built upon our previous models, Materials Studio molecular dynamics (MD) software was used to simulate the nucleation of an $84.183 \AA$ diameter water droplet, consisting of 1705 water molecules. This water droplet was initially created from a pre-constructed ice crystal from the Materials Studio library, which was then melted to form a water droplet. This was accomplished within Materials Studio by using the Forcite Dynamics NVT function (constant number of particles- $n$, volume $-\mathrm{V}$, and temperature- $\mathrm{T}$ ) at $373 \mathrm{~K}$ for 5000 picoseconds (ps). The forcefield used was COMPASS II, and the interaction distance for hydrogen bonds was set to $19 \AA$, allowing the surrounding water molecules to coalesce. The resulting water droplet is identical to the one used in our previous simulations and is able to fully nucleate and re-crystallize within 10,000 picoseconds (ps) upon re-cooling. This timescale was therefore used as a baseline for determining the effects of the various compounds and interactions on the water droplet. Additionally, to ensure consistency and reproducibility, the molecular dynamics simulation of the nucleating water droplet was run several times. Each run was found to be consistent in regard to the onset of nucleation and subsequent crystallization-thus, this model was 
used as the baseline for all nucleation models in this study, including pure water, and the models containing unmodified and modified AFGP compounds.

A base AFGP structure was manually constructed in Materials Studio with no modifications to the hydroxyl groups (in blue), as shown in Figure 1a. This original AFGP structure was constructed with four repeating peptide units, to mimic the structure of native AFGP-8; however, no modifications were made to the peptide structure to eliminate any effects of the chain and to focus solely on the saccharide moieties. From this structure, three variations were produced. For each variation, the hydroxyl groups on the saccharide structures were modified by either shortening or lengthening the distances between the hydroxyl groups and the saccharide structure by adding or eliminating carbon atoms. Three modified variations of the AFGP structure were constructed manually, forming modified AFGP (1), (2), and (3), as shown in Figure 1b-d, respectively. In the modified AFGP compounds, unchanged hydroxyl groups are shown in black, while modified hydroxyl groups are shown in red. Each hydroxyl group was given an identification number, between 1 and 6, also shown in red.

\section{a) Unmodified AFGP}

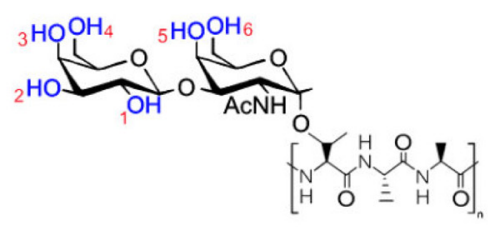

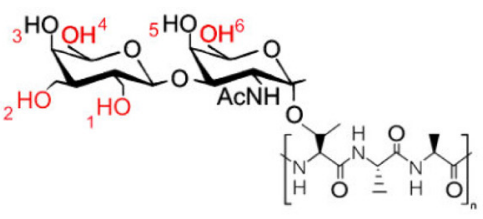

b) Modified AFGP (1)

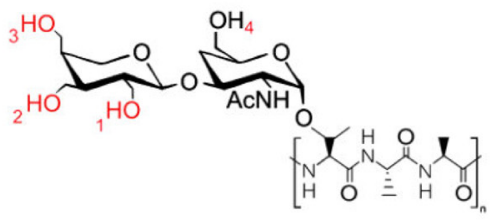

c) Modified AFGP (2)

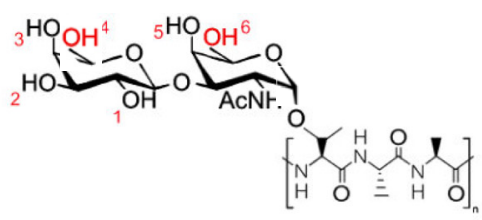

d) Modified AFGP (3)

Figure 1. AFGP structures, modeled in this study. The original unmodified AFGP structure depicts the original locations of the hydroxyl groups in blue, and (b) modified AFGP (1), (c) modified AFGP (2), and (d) modified AFGP (3). Altered hydroxyl groups are shown in red.

After construction, each AFGP compound was optimized for geometry using the Materials Studio Geometry Optimization function for 5000 ps using the Newton-Raphson method within the structure optimization module. Distances were then measured by the shortest direct distance between hydrogens, using the distance tool in Materials Studio. The distances between the hydrogen atoms of the corresponding hydroxyl groups were measured after geometry optimization was performed and after the structures were fully relaxed, but before the AFGP structures were placed in contact with the water droplet. Because of the repeating saccharide units, the measured distances were averages of all the hydroxyl group distances on all the saccharide structures. For all unmodified and modified AFGP structures, hydroxyl distances were measured only between the closest adjacent groups on the same saccharide structures. Therefore, only the distances between hydroxyl groups 1-2, 2-3, 3-4, and 5-6 were measured, if applicable.

As previously investigated, hydroxyl distances of less than $2.858 \AA$, but greater than $7.117 \AA$, were found to affect ice nucleation. Thus, in this study, hydroxyl group distance modifications were conducted to fit as close to this range as possible. As such, while the hydroxyl distances of unmodified AFGP fit neatly into the range of 2.858-7.117 $\AA$, modified AFGP structures were made with hydroxyl distances that were approximately as close to either $3.0 \AA$ or $7.0 \AA$ as possible. Additionally, the modified AFGP structures were 
constructed with some variability in the amount of modified hydroxyl group structures, thus modified AFGP (1) contains four modified hydroxyl groups, AFGP (2) contains three modified hydroxyl groups, and AFGP (3) contains two. The measured distances between adjacent hydroxyl groups on the unmodified AFGP compound are shown in Table 1.

Table 1. Hydroxyl distances for unmodified and modified AFGP compounds (1), (2), and (3).

\begin{tabular}{ccccc}
\hline \multirow{2}{*}{ Compound } & \multicolumn{4}{c}{ Adjacent Hydroxyl Distances (OH Groups) } \\
& $\mathbf{1 - 2}$ & $\mathbf{2 - 3}$ & $\mathbf{3 - 4}$ & $\mathbf{5 - 6}$ \\
\hline Unmodified AFGP & $3.449 \AA$ & $3.506 \AA$ & $4.267 \AA$ & $5.518 \AA$ \\
Modified AFGP (1) & $4.331 \AA$ & $4.749 \AA$ & $3.105 \AA$ & $2.813 \AA$ \\
Modified AFGP (2) & $7.327 \AA$ & $6.160 \AA$ & $\mathrm{N} / \mathrm{A}$ & $\mathrm{N} / \mathrm{A}$ \\
Modified AFGP (3) & $3.502 \AA$ & $4.997 \AA$ & $2.792 \AA$ & $2.715 \AA$ \\
\hline
\end{tabular}

For modified AFGP (1), hydroxyl groups 1, 2, 4, and 6 were modified, as shown in Table 1. For modified AFGP (2), two hydroxyl groups were removed from AFGP (2), leaving a total of four hydroxyl groups on the saccharide structures. The remaining hydroxyl groups were modified, and distances were increased, as shown in Figure $1 \mathrm{~b}$. The distances between the remaining hydroxyl groups were, therefore, $7.327 \AA$ and $6.060 \AA$ between hydroxyl groups 1-2 and 2-3, respectively.

For modified AFGP (3), hydroxyl groups 4 and 6 were modified by removing a carbon atom, moving them closer to the saccharide structures. Therefore, the measured distance between hydroxyl groups 3-4 and 5-6 were $2.792 \AA$ and $2.715 \AA$, respectively. Changes in the remaining hydroxyl distances are shown in Table 1.

This study utilized the COMPASS II forcefield for all nucleation simulations, which were run using Forcite Dynamics. In Materials Studio, the Forcite Dynamics module controls the classic molecular dynamics simulations. For all simulations, the bonding distance was set to $19 \AA$, and all nucleation simulations were run at standard atmospheric pressure. Similar to the methods used in our previous work, the nucleation simulations were run first at NVE (constant number of particles N, volume V, and energy E) for 5000 picoseconds (ps). This was followed by NVT for a total of 15,000 picoseconds (ps), and the temperature was held constant at 250 K. Quasi-Newton-Raphson was used for all Forcite Dynamics simulations of the water droplet. Simulations of the water droplet were conducted first, followed by nucleation simulations of unmodified AFGP, to provide a baseline comparison.

For models containing AFGP compounds, the unmodified and modified AFGP structures were placed manually next to the water droplet, at a distance of $12 \AA$, and all the structures were unconstrained and allowed to freely move during the simulation. Then the entire model, containing both the water droplet and a single AFGP compound, was again optimized for geometry before running molecular dynamics.

This study thus evaluated ice nucleation in terms of free energy, which was used to determine the onset and duration of crystallization. Previous studies, such as Matsumoto et al., have evaluated the free energy profile to determine the crystallization of a nucleus. Matsumoto et al., showed that the system's potential energy can be used as an indicator of nucleation, due to the initial nucleating crystal expanding rapidly, forming three dimensional hexagonal rings [19]. The system's energy thus decreases quickly as the surrounding water molecules surround the growing nucleus and anchor into place, and 'solidify' within the ice crystal [19]. As a result, the final crystallized ice structure has lower potential energy compared to water.

This method is also based upon the classical nucleation theory (CNT), for which the equation for the Gibbs energy barrier $G$ of a spherical cluster consisting of $N$ particles can be expressed by [25]:

$$
\Delta \mathrm{G}(\mathrm{N})=-\mathrm{N} \Delta \text { fus } \mu+\gamma[36 \pi(\mathrm{N} / \rho) 2] 1 / 3
$$


where $\rho$ is the number density of the crystalline phase, $\gamma$ is the interfacial free energy between the two phases, and $\Delta$ fus $\mu$ is the change in chemical potential on fusion. Therefore, for numerical ice nucleation systems, CNT can be obtained [25].

Thus, all nucleation simulations were monitored for changes in potential energy, and the total potential energy of each simulation was recorded before, during, and after nucleation as a means to indicate the onset and termination of ice nucleation and crystallization. In order to visually observe the onset, duration, and completion of nucleation, the energies were plotted as a function of time (ps).

\section{Results}

All ice nucleation results are shown in Figure 2, plotted as a function of potential energy vs. time (ps). Ice nucleation was assumed to occur when the total potential energy began to consistently decrease at a steady rate. Complete crystallization was observed by the energy of the system remaining constant, which was confirmed by visual observation of the fully crystalline structure.

The simulation containing the pure water droplet saw an initiation of nucleation at 2970 ps, which was identical to our previous simulation results (Figure 2a). However, the model containing the water droplet with unmodified AFGP saw no changes in potential energy of the system, and neither ice nucleation nor crystallization occurred.

Unlike the unmodified AFGP compound, modified AFGP compounds (1) and (2) saw a drop in potential energy, indicating ice nucleation and crystallization. Ice nucleation for modified AFGP (1) was delayed, initiating at 2105 ps, followed by a steep decline in the total potential energy of the system and rapid crystallization, which terminated at 6200 ps. By contrast, the onset of ice nucleation for the water droplet model containing modified AFGP (2) was immediate, occurring directly at the beginning of the simulation $(0 \mathrm{ps})$ and continuing until crystallization was complete, which occurred at $8100 \mathrm{ps}$ (Table 2). Variations in the initiation and termination of crystallization of all the molecular dynamics models are shown in Table 2.

The molecular dynamics model containing modified AFGP (3) experienced rapid swings in potential energy for the first 2000 ps, which gradually decreased until reaching the final potential energy of the system of $-62342 \mathrm{~kJ} / \mathrm{mol}^{-1}$. While only a gradual decrease in total potential energy was observed, crystallization did occur, initiating at 1900 ps.

As shown in Figure 2a-e, the unmodified AFGP saw the smallest decline in potential energy, while the initial and final potential energies for pure water, modified AFGP (1) and (3) were similar in their start and endpoints, as well as the degree of decline in potential energy (Figure 2c,e). By contrast, modified AFGP (2) saw the most significant decline, from $-52,156$ to $-69,776 \mathrm{~kJ} / \mathrm{mol}^{-1}$ (Figure 2d). 

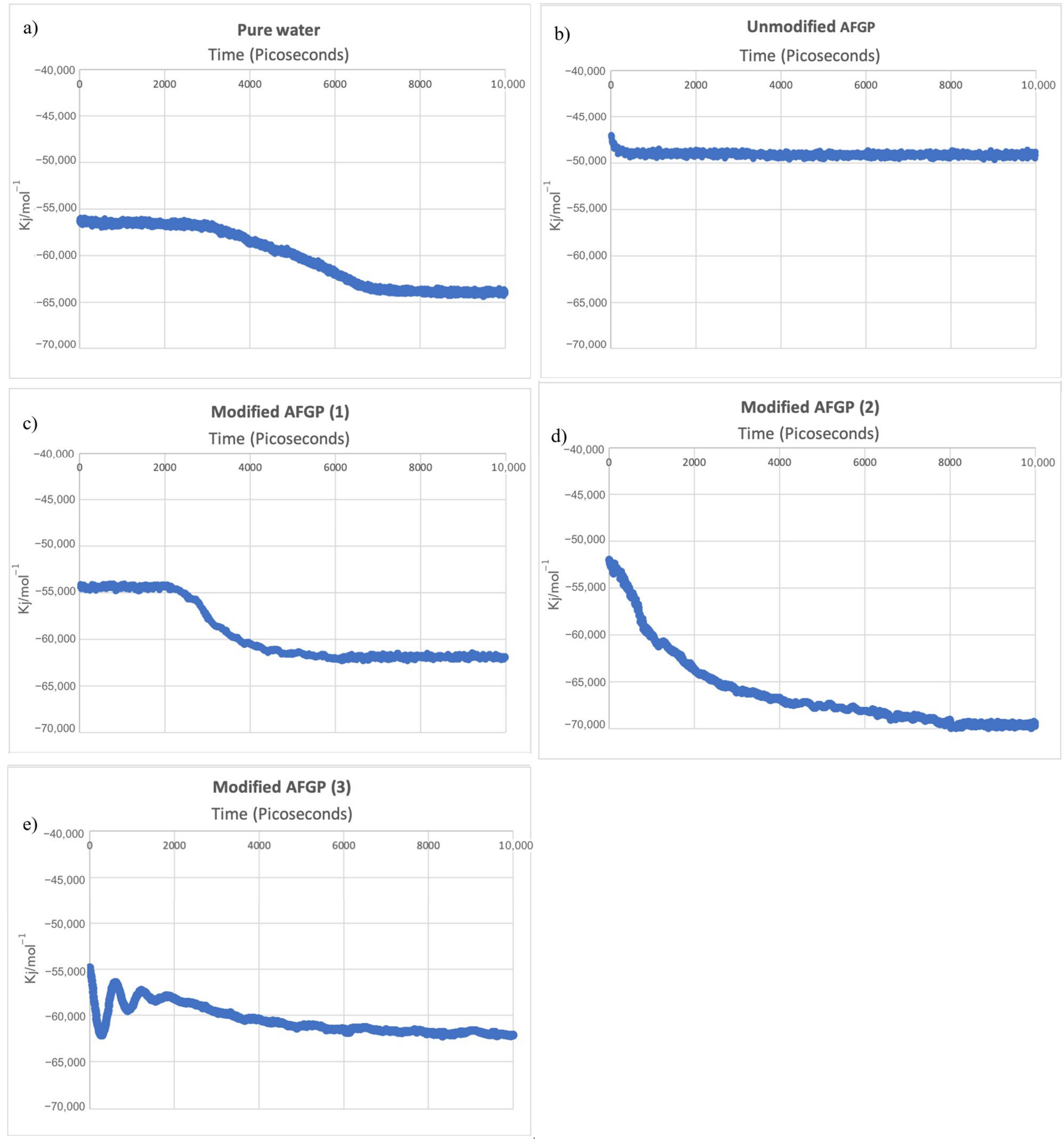

Figure 2. The total potential energy of the molecular dynamics models indicating ice nucleation of (a) pure water, (b) unmodified AFGP, (c) modified AFGP (1), (d) modified AFGP (2), and (e) modified AFGP (3). 
Table 2. Initiation and termination of crystallization of all simulated molecular dynamics models that successfully crystallized in this study.

\begin{tabular}{ccc}
\hline Compound & Initiation of Crystallization & $\begin{array}{c}\text { Termination of Crystallization } \\
\text { Picoseconds (ps) }\end{array}$ \\
\hline Pure water & 2970 & 6900 \\
Unmodified AFGP & - & - \\
Modified AFGP (1) & 2105 & 6200 \\
Modified AFGP (2) & 0 & 8100 \\
Modified AFGP (3) & 1900 & 8200 \\
\hline
\end{tabular}

\section{Discussion}

Molecular dynamics simulations show the simulated pure water droplet crystallized within the allotted simulation time, albeit after a brief delay. This model was used as a baseline for assessing the water droplet crystallization times and total potential energies of the system of the unmodified and modified AFGP compounds. As shown by the changes in the potential energies of the modeled molecular dynamics systems, all modified AFGP compounds resulted in the crystallization of the water droplet. By contrast, simulation of the water droplet with the unmodified AFGP compound did not crystalize within the allotted time of 10,000 ps, and mostly maintained its total potential energy throughout. This confirmed the antifreeze activity of the native AFGP compound within the simulation. Additionally, this supports the literature studies, which have found that the number of hydroxyl groups may be less critical to antifreeze activity compared to the specific conformation of the structures.

All three of the modified AFGP compounds resulted in the crystallization of the water droplet, with some similarities. Specifically, the pure water and modified AFGP (1) and (3) simulations resulted in similar initial and final potential energies following crystallization (Figure 2). Additionally, there was a similar time delay in ice nucleation and crystallization of pure water and modified AFGP (1), as pure water initiated at approximately 2970 ps, while AFGP (1) initiated at approximately 2105 ps (Table 2). By contrast, modified AFGP (2) initiated immediately at 0 ps. Similarly, modified AFGP (3) also experienced a time delay in the initiation of ice crystallization, however, due to the sporadic nature of the potential energy curve for AFGP (3), it was difficult to ascertain the exact onset of ice nucleation. The initiation of nucleation, therefore, was estimated based upon visual observation of the molecular dynamics simulations, wherein the onset of ice nucleation began at approximately 1900 ps.

The total time of crystallization of pure water (3930 ps), was similar to that of AFGP (1) (4095 ps), which was in contrast to the crystallization times of AFGP (2) and AFGP (3), which were both significantly longer in comparison (Table 2). This may indicate that the modifications of the hydroxyl group separation distances between groups 3-4 and 5-6, specifically, did result in changes in antifreeze activity, removing the AFGP compound's ability to prevent ice crystallization. In modified AFGP compounds (1) and (3), the hydroxyl group separation distances of 3-4 and 5-6 were all close to, or smaller than, $\sim 3.1 \AA$, which is in the range for promotion of nucleation per our previous work. In comparison, the unmodified native AFGP structure had hydroxyl group separation distances for hydroxyl groups 3-4 and 5-6 of $4.267 \AA$ and $5.518 \AA$, respectively, which is in the range of inhibition of nucleation.

The rapid swings observed in the potential energy of the system for the AFGP (3) model may be due to a few possible factors. Because the AFGP (3) structure is the only molecule without a methanol group off the external ring (the intermediate carbon), we believe that the presence of symmetry on this ring allows the whole ring structure to rotate, or spin, with the bond to the ether oxygen as a pivot point. The magnitude of this motion may decrease with decreasing temperature (kinetic energy) and stabilize as a configuration with the lowest potential energy is reached. 
The presence of the AFGP (3) compound may also be inadvertently affecting the water droplet as it begins to nucleate. A localized nucleation point may be briefly allowing some water molecules to stabilize, thus briefly lowering the potential energy of the system. However, the movement of the AFGP (3) structure may disrupt this process, melting the aforementioned nucleus and raising the potential energy as the water molecules move again. This cycle appears to repeat, before finally stabilizing, nucleating, and lowering the overall potential energy as the system completely crystalizes.

Furthermore, the modified AFGP (3) compound resulted in the elongation of the water droplet, right before the onset of ice crystallization (Figure 3). This may be partially explained by the structure of the compound, due to the loss of the carbon between the oxygen and the saccharide ring in groups 3-4 and 5-6, resulting in more interactions between the oxygen the ring due to sharing electron density and splitting protons. Therefore, the hydrogens were able to interact with each other as well. This increased electron localization in the ring, and its electron orbital field, may result in a transitory field that causes the water droplet to respond, initially repulsively, to the shape of the AFGP molecule. As atoms slow down, due to temp, the repulsion may decrease, becoming more thermodynamically stable. This was not observed in the modified AFGP (1) structure, as hydroxyl groups 1-2 had carbons inserted, which pushed the oxygen atoms out, offsetting the effects of the groups 3-4 being moved closer to the saccharide ring.

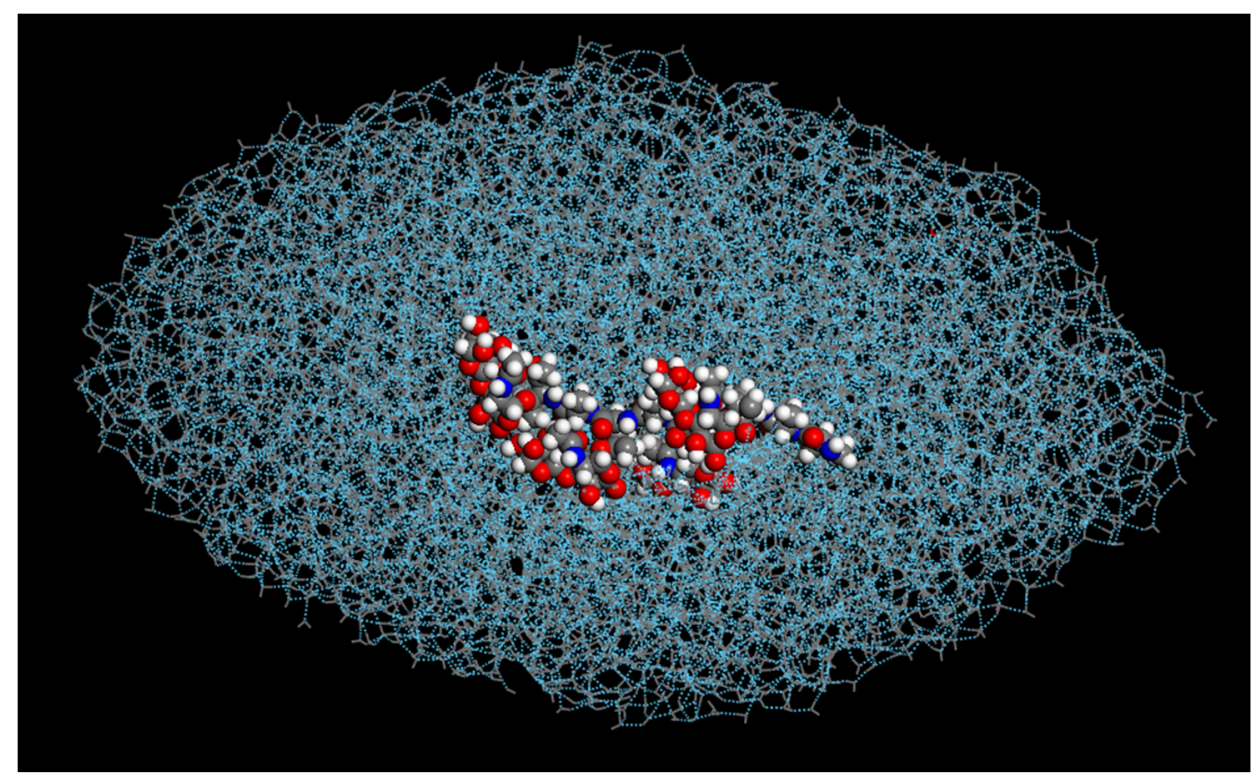

Figure 3. Modified AFGP (3) and oval water droplet model at approximately 1400 ps, before the onset of crystallization.

As all of the AFGP compounds were not restricted during the molecular dynamics simulations, they were allowed to freely move during the simulations. Upon completion of crystallization, all of the modified AFGP compounds adhered to the primary prism plane (1010) of the ice crystal, as shown in Figure 4, where the modified AFGP (3) compound adhered to the edge of the primary prism plane of the fully crystallized water droplet. Studies have found that standard AFGP compounds preferentially adhere to the primary prism plane, as a means of preventing further ice nucleation; thus, this property appears to have been retained in the modified AFGP compounds, though ice nucleation and crystallization was not prevented.

Additionally, the effects of modified AFGP (2) on the ice crystallization of the water droplet appear to be different from the modified AFGP (1) and (3) structures. The modified AFGP (2) compound had only two adjacent hydroxyl groups, with separation distances of $7.327 \AA$ and $6.160 \AA$ for groups 1-2 and 2-3, respectively. The simulation AFGP (2) showed immediate crystallization, however, indicating possible promotion of ice nucleation and 
crystallization. Moreover, our previous results indicated that compounds with hydroxyl group separation distances less than $2.858 \AA$, but greater than $7.117 \AA$ were correlated with the promotion of ice nucleation and crystallization, and the results from modified AFGP (2) suggest that activity may occur closer to $6.160 \AA$ as well.

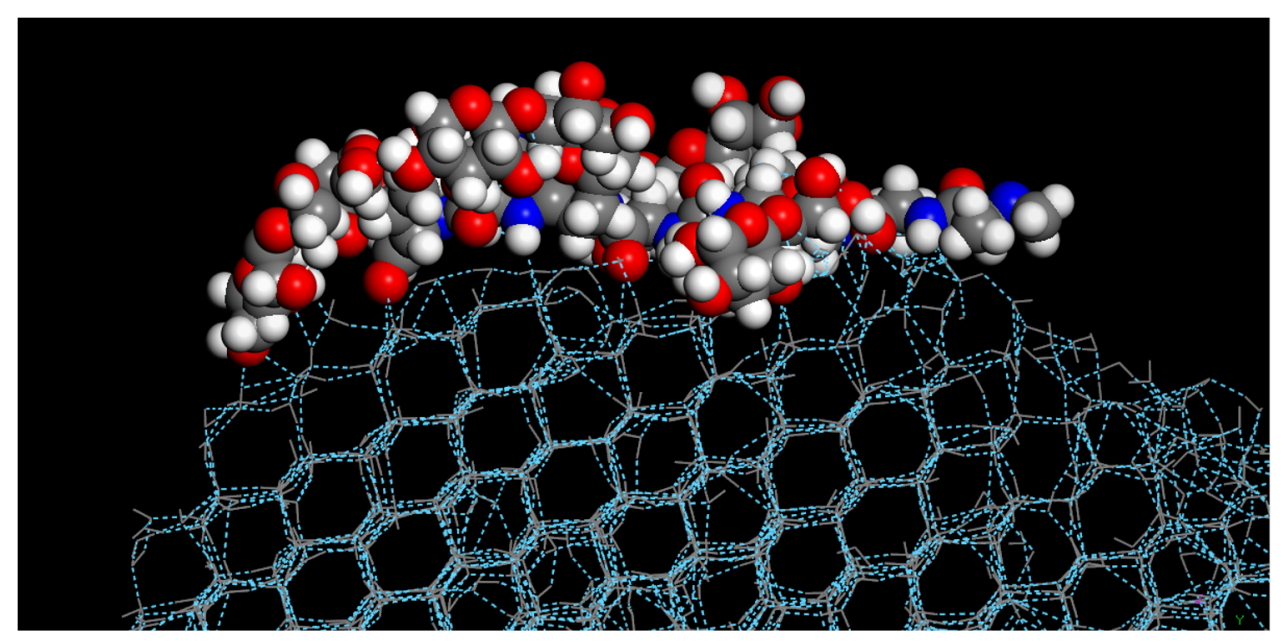

Figure 4. Interaction of modified AFGP (3) with completely the crystallized water droplet after molecular dynamics simulation.

While modification of the hydroxyl groups resulted in water droplet crystallization, the reasons for the observed differences in ice crystallization between pure water and the modified AFGP compounds remain unclear. It is possible that the bond angles for the relevant hydroxyl groups play a role, as when measured before nucleation simulations, there was some variability. For example, for modified AFGP (3), the hydroxyl group bond angles ranged between $108.8^{\circ}$ and $110.2^{\circ}$. By contrast, hydroxyl group bond angles for AFGP (1) ranged between $106.4^{\circ}$ to $114.6^{\circ}$. However, further study will be needed to better understand what effects hydroxyl group bond angle may have on antifreeze activity.

It is also possible that the electronegativity of the oxygen atom in each group is affected by the proximity of the saccharide ring, which ultimately affects the rotation and location of the hydrogen atoms. The hydrogen atoms may be potentially drawn closer to the oxygen atom in unmodified AFGP, as the oxygen atoms are more electronegative (farther from the ring). This could indicate that structural arrangements of groups 3-4 and 5-6 are critical to inhibition, which requires an ideal geometry for the inhibition, or promotion, of nucleation to occur.

While the modified AFGP compound followed our previous findings, losing its ability to prevent ice crystallization when the hydroxyl groups were modified with distances smaller than $\sim 3.0 \AA$ and larger than $\sim 7.0 \AA$, there are still some notable limitations to this study. Evaluation of hydroxyl group modifications using a step-by-step approach to assess the importance of each hydroxyl group distance will help to better understand if specific hydroxyl groups are critical for ice nucleation prevention. Additionally, the peptide backbone is known to play a role in the activity of AFGPs, though its exact mechanism is still unclear. Assessing peptide backbone modifications would help to better understand its functional role in comparison to the effects of the saccharide structures, though this was not assessed in this study.

Because this study assessed only a narrow hydroxyl distance range to coincide with our previous work, additional models will also need to be considered for test a variety of hydroxyl modifications, such as single hydroxyl distance modifications, and a wider range of distances. These considerations will be assessed in our future numerical work.

Furthermore, while the molecular dynamics simulations in this study support the notion that hydroxyl group distance requires a specific range for ice crystallization inhibition to occur, an experimental assessment would provide additional verification. Comparing 
native AFGP and modified AFGP compounds for ice nucleation prevention activity would ideally show the real-life effects of hydroxyl group distance modification. Some studies, such as Budke et al., have already shown that native AFGPs are the most effective ice nucleation inhibition agents [26]. Additionally, the researchers showed that the effectiveness of modified AFGPs is strongly reduced when in the form of monosaccharide AFGP analogs, which reduced the number of hydroxyl groups in the overall structure [26]. Though experimental modification of AFGPs has been fraught with some difficulty in the past, new techniques, such as targeted sequence modification of synthetic AFGPs, as done by Nagel et al., may provide an avenue for targeted hydroxyl group modification [27]. This will also be explored in our future work.

\section{Conclusions}

AFGP compounds are effective against preventing ice crystallization, and their saccharide structures play a key role in ice nucleation inhibition. This study investigated the effects of AFGP compounds with various hydroxyl group separation distances using molecular dynamics simulations. The models supported the notion that AFGP saccharide structures play a key role in preventing ice crystallization, and that modifications to the hydroxyl groups result in the loss of antifreeze activity. Specifically, modified AFGP structures containing hydroxyl distances smaller than $\sim 3.0 \AA$ lost their ability to prevent ice crystallization, while the AFGP compound containing hydroxyl distances of $7.327 \AA$ and $6.160 \AA$ was correlated a promotion in ice nucleation. However, further numerical and experimental investigations into the effects of saccharide modification will need to be conducted to determine which hydroxyl groups are more susceptible to change in hydroxyl group separation distance, and distance from the saccharide structure itself.

Author Contributions: Conceptualization, M.B.; methodology, M.B.; software, M.B.; validation, M.B.; formal analysis, M.B., M.R.; investigation, M.B.; data curation, M.B.; writing-review and editing, M.B., M.R.; project administration, M.B. All authors have read and agreed to the published version of the manuscript.

Funding: This research was funded by National Science Foundation I/UCRC Center for Novel High Voltage Materials and Structures under \#IIP 1362135 and by the NSF Grant Opportunities for Academic Liaison with Industry program under \#CMMI-123252, and The Colorado Office of Economic Development and International Trade, Grant Award \#37977.

Data Availability Statement: The authors confirm that the data supporting the findings of this study are available within the article.

Conflicts of Interest: The authors declare no conflict of interest.

\section{References}

1. Devries, A.L. Glycoproteins as Biological Antifreeze Agents in Antarctic Fishes. Science 1971, 172, 1152-1155. [CrossRef]

2. Harding, M.M.; Anderberg, P.I.; Haymet, A.D.J. 'Antifreeze' glycoproteins from polar fish. JBIC J. Biol. Inorg. Chem. 2003, 270, 1381-1392. [CrossRef]

3. Geoghegan, K.; Osuga, D.; Ahmed, A.; Yeh, Y.; Feeney, R. Antifreeze glycoproteins from Polar fish. Structural requirements for function of glycopeptide 8. J. Biol. Chem. 1980, 255, 663-667. [CrossRef]

4. Knight, C.; Driggers, E.; Devries, A. Adsorption to ice of fish antifreeze glycopeptides 7 and 8. Biophys. J. 1993, 64, $252-259$. [CrossRef]

5. Meister, K.; Devries, A.L.; Bakker, H.J.; Drori, R. Antifreeze Glycoproteins Bind Irreversibly to Ice. J. Am. Chem. Soc. 2018, 140, 9365-9368. [CrossRef]

6. Mochizuki, K.; Molinero, V. Antifreeze Glycoproteins Bind Reversibly to Ice via Hydrophobic Groups. J. Am. Chem. Soc. 2018, 140, 4803-4811. [CrossRef] [PubMed]

7. Urbańczyk, M.; Góra, J.; Latajka, R.; Sewald, N. Antifreeze glycopeptides: From structure and activity studies to current approaches in chemical synthesis. Amino Acids 2016, 49, 209-222. [CrossRef] [PubMed]

8. Meister, K.; Moll, C.J.; Chakraborty, S.; Jana, B.; Devries, A.L.; Ramløv, H.; Bakker, H.J. Molecular structure of a hyperactive antifreeze protein adsorbed to ice. J. Chem. Phys. 2019, 150, 131101. [CrossRef]

9. Voets, I.I. From ice-binding proteins to bio-inspired antifreeze materials. Soft Matter 2017, 13, 4808-4823. [CrossRef] [PubMed] 
10. Wierzbicki, A.; Taylor, M.S.; Knight, C.A.; Madura, J.D.; Harrington, J.P.; Sikes, C.S. Analysis of shorthorn sculpin antifreeze protein stereospecific binding to (2-1 0) faces of ice. Biophys. J. 1996, 71, 8-18. [CrossRef]

11. Ahn, M.-J.; Murugan, R.N.; Kim, E.-J.; Lee, J.-H.; Cheong, C.-J.; Kang, S.-W.; Park, H.-J.; Shin, S.-Y.; Kim, H.-J.; Bang, J.-K. Studies on the Effect of Number of Sugar Moiety in the Antifreeze Activity of Homodimeric AFGPs. Bull. Korean Chem. Soc. 2012, 33, 2411-2414. [CrossRef]

12. Tachibana, Y.; Fletcher, G.L.; Fujitani, N.; Tsuda, S.; Monde, K.; Nishimura, S.-I. Antifreeze Glycoproteins: Elucidation of the Structural Motifs That Are Essential for Antifreeze Activity. Angew. Chem. 2004, 116, 874-880. [CrossRef]

13. Surís-Valls, R.; Voets, I.K. Peptidic antifreeze materials: Prospects and challenges. Int. J. Mol. Sci. 2019, 20, 5149. [CrossRef]

14. Peltier, R.; Evans, C.W.; Devries, A.L.; Brimble, M.A.; Dingley, A.J.; Williams, D.E. Growth Habit Modification of Ice Crystals Using Antifreeze Glycoprotein (AFGP) Analogues. Cryst. Growth Des. 2010, 10, 5066-5077. [CrossRef]

15. Liu, S.; Ben, R.N. C-linked galactosyl serine AFGP analogues as potent recrystallization inhibitors. Org. Lett. 2005, 7, 2385-2388. [CrossRef] [PubMed]

16. Anand, N.K.; Holm, C.; Smiatek, J. Local water dynamics around antifreeze protein residues in the presence of osmolytes: The importance of hydroxyl and disaccharide groups. J. Phys. Chem. B 2014, 118, 11613-11621.

17. Pandey, P.; Mallajosyula, S.S. Elucidating the role of key structural motifs in antifreeze glycoproteins. Phys. Chem. Chem. Phys. 2019, 21, 3903-3917. [CrossRef]

18. Bleszynski, M.; Reil, M.; Kumosa, M. Hydroxyl Group Separation Distances in Anti-Freeze Compounds and Their Effects on Ice Nucleation. Int. J. Mol. Sci. 2020, 21, 8488. [CrossRef]

19. Matsumoto, M.; Saito, S.; Ohmine, I. Molecular dynamics simulation of the ice nucleation and growth process leading to water freezing. Nature 2002, 416, 409-413. [CrossRef]

20. Nandi, P.K.; Burnham, C.J.; English, N.J. Electro-nucleation of water nano-droplets in No Man's Land to fault-free ice Ic. Phys. Chem. Chem. Phys. 2018, 20, 8042-8053. [CrossRef]

21. Fitzner, M.; Philipp, P.; Michaelides, A. Predicting heterogeneous ice nucleation with a data-driven approach. Nat. Commun. 2020, 11, 4777. [CrossRef] [PubMed]

22. Röttger, K.; Endriss, A.; Ihringer, J.; Doyle, S.; Kuhs, W.F. Lattice constants and thermal expansion of $\mathrm{H}_{2} \mathrm{O}$ and $\mathrm{D}_{2} \mathrm{O}$ ice $\mathrm{Ih}$ between 10 and 265 K. Acta Crystallogr. Sect. B Struct. Sci. 1994, 50, 644-648. [CrossRef]

23. Czechura, P.; Tam, R.Y.; Dimitrijevic, E.; Murphy, A.V.; Ben, R.N. The Importance of Hydration for Inhibiting Ice Recrystallization with C-Linked Antifreeze Glycoproteins. J. Am. Chem. Soc. 2008, 130, 2928-2929. [CrossRef] [PubMed]

24. Nada, H.; Furukawa, Y. Antifreeze proteins: Computer simulation studies on the mechanism of ice growth inhibition. Polym. J. 2012, 44, 690-698. [CrossRef]

25. Reinhardt, A.; Doye, J.P.K. Free energy landscapes for homogeneous nucleation of ice for a monatomic water model. J. Chem. Phys. 2012, 136, 054501. [CrossRef]

26. Budke, C.; Dreyer, A.; Jaeger, J.; Gimpel, K.; Berkemeier, T.; Bonin, A.S.; Nagel, L.; Plattner, C.; Devries, A.L.; Sewald, N.; et al. Quantitative Efficacy Classification of Ice Recrystallization Inhibition Agents. Cryst. Growth Des. 2014, 14, 4285-4294. [CrossRef]

27. Nagel, L.; Plattner, C.; Budke, C.; Majer, Z.; Devries, A.L.; Berkemeier, T.; Koop, T.; Sewald, N. Synthesis and characterization of natural and modified antifreeze glycopeptides: Glycosylated foldamers. Amino Acids 2011, 41, 719-732. [CrossRef] 